\title{
Optimised Combinatorial Control Strategy for Active Anti-Roll Bar System for Ground Vehicle
}

\author{
N. Zulkarnain ${ }^{1,4}$, H. Zamzuri ${ }^{2}$, S. A. Saruchi ${ }^{2}$, A. Hussain ${ }^{1}$, S. S. Mokri ${ }^{1}$, A. Jedi ${ }^{3}$, N. Razali ${ }^{3,4}$ and I. N. A. Mohd \\ Nordin $^{5}$ \\ ${ }^{1}$ Centre for Integrated Systems Engineering and Advanced Technologies (INTEGRA), Faculty of Engineering and Built Environment, \\ Universiti Kebangsaan Malaysia \\ ${ }^{2}$ Vehicle System Engineering, Malaysia-Japan International Institute of Technology, Universiti Teknologi Malaysia \\ ${ }^{3}$ Centre of Integrated Design for Advanced Mechanical Design (PRISMA), Faculty of Engineering and Built Environment, Universiti \\ Kebangsaan Malaysia \\ ${ }^{4}$ Centre of Research in Engineering Education and Built Environment (PEKA), Faculty of Engineering and Built Environment, Universiti \\ Kebangsaan Malaysia \\ ${ }^{5}$ Department of Electrical Engineering Technology, Faculty of Engineering Technology, Universiti Tun Hussein Onn Malaysia \\ *Corresponding author E-mail: shikinzulkarnain@ukm.edu.my
}

\begin{abstract}
The objective of this paper is to optimise the proposed control strategy for an active anti-roll bar system using non-dominated sorting genetic algorithm (NSGA-II) tuning method. By using an active anti-roll control strategy, the controller can adapt to current road conditions and manoeuvres unlike a passive anti-roll bar. The optimisation solution offers a rather noticeable improvement results compared to the manually-tuned method. From the application point of view, both tuning process can be used. However, using optimisation method gives a multiple choice of solutions and provides the optimal parameters compared to manual tuning method.
\end{abstract}

Keywords: anti-roll bar; ride; handling; control strategy; optimisation.

\section{Introduction}

The growing market share of light-duty vehicles has prompted automotive engineers to examine body roll minimisation strategies $[1,2]$. During driving manoeuvres in high-centre-of-gravity vehicles, due to its roll-over, dangerous operating scenarios may induce drivers to drive in an aggressive way, engaging in driving behaviours such as high lateral acceleration, rapid tire dilation and emergency lane change in $[3,4]$. Commercially, to counteract the roll movement, the most used topology is the use of an anti-roll bar. This bar can be implemented in passive and active topologies, where some of the most important parameters performance, energy consumption and costs [3].

A passive anti-roll bar is also known as a stabiliser bar, which usually is a U-shaped rod which is bound parallel to the ground. It is also known as torsion spring, where a component that springs back when twisted. Most often, this bar is employed on independent front suspensions. The control arms rise simultaneously on both sides when the vehicle drives over a bump. Both ends of the anti-roll bar turn upward with them, and the bar does not influence the behaviour. However, the control arms on the outside lifts the end of the bar and the opposite control arm pulls its end down during manoeuvre. The anti-roll bar reduces the movement of the control arms and minimises body leaning by resisting and twisting this torsion.

A disadvantage of this passive anti-roll bar is the coupling between the left and right side of the vehicle. For example, when a vehicle driving in a straight line hits a bump with only one side of the vehicle, the passive anti-roll bar is twisted by the control arms, and its resistance to this movement results in the other wheel being lifted, reducing road contact and furthermore transferring the bumping effect to the vehicle body, thus reducing the passenger comfort. This effect is worse when driving during cornering; the inner wheel hits a bump, reducing the degree of twisting of the anti-roll bar and thus the vertical force on the outer wheel, possibly resulting in loss of control.

Active anti-roll bars are used to overcome the drawback of the passive anti-roll bars, in which a linear or rotary actuator acts in series with a conventional passive anti-roll bar to provide forces that resist vehicle roll. Under normal driving condition, these systems reduce anti-roll force and road impact by lowering the hydraulic pressure in the actuators, thus decoupling the left and right side. During cornering, the actuators are pressurised, applying the required anti-roll bar force to maintain near-zero body roll angle. This is achieved by real-time response to actual or anticipated driving conditions using the centre-of-gravity lateral accelerometers and vertical lateral accelerometers [5].

Various methods and approaches are used to design and active anti-roll bar systems. Each method has its own advantages and disadvantages. Research on active anti-roll bar system is mainly covers the ride and handling performance tests. Most researchers have been used conventional controllers to control an active antiroll bar system; these controllers are Proportional Integral (PI) and Proportional Integral Derivative (PID), which have advantages in fast tracking application but have a large overshoot. For example, the author in [6-8] addressed the problem by using conventional controllers. Some authors implemented the modern controllers such as LQ controller and predictive controller, which only used 
trial-and-error tuning method without applying a complex tuning method to optimally design the controller [9-11]. Given these disadvantages, the proposed control method, namely, composite nonlinear feedback and linear quadratic Gaussian (CNF-LQG) fusion control strategy is developed to improve settling time and to reduce the overshoot. The parameters of this control method are optimised by using NSGA-II tuning method [12].

\section{Combinatorial Control Strategy}

A new concept that combines CNF and LQG controllers emerged from an analysis of the CNF and LQG control strategies for an active anti-roll bar system. The CNF controller can overcome achieve fast response without overshoot and involves some mathematical derivation and basic control theory, while the LQG controller provides optimal control. Therefore, the CNF-LQG fusion control strategy is used to improve the responses for both handling and ride comfort performance. The objective of the active ARB controller is to reject disturbance and to improve the roll angle and roll rate responses as close to zero. The controllers used in this section are the CNF and LQG controllers. These controllers are designed and tuned to generate the input of torque to control the active ARB system [13]. Figure 1 shows a block diagram configuration of combination of CNF controller, LQR controller, and Kalman filter estimator to form a compensator. This solution in designing the LQG controller is based on the separation principle where the LQR controller and Kalman filter are designed independently and then combined to form the LQG compensator. A Kalman filter is a typical application and a feasible estimation approach that can fuse multiple sensory measurements to provide an accurate position estimation.

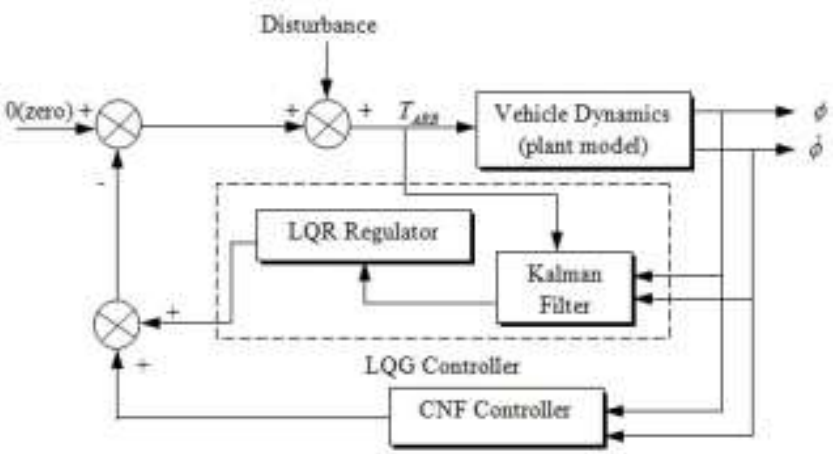

Fig. 1: CNF_LQG fusion block diagram configuration

The CNF controller has two parts namely a linear feedback part and nonlinear feedback part. The objective of linear feedback is to obtain a small damping ratio for quick response, and nonlinear feedback is designed to increase the damping ratio as the system output approaches the target reference to avoid overshoot. In this study, the target reference is zero. The linear control law for torque of anti-roll bar is defined as in (1):

$T_{A R B}=$ Disturbance $-K_{l q r} \dot{\hat{x}}+F_{x}+G r+\rho(y, r) B^{T} P\left(x-x_{e}\right)\left[\begin{array}{c}\phi \\ \dot{\phi}\end{array}\right]$

where

$$
\begin{aligned}
& K_{l q r}=R_{l q r} B^{T} P_{l q r}, \dot{\hat{x}}=\left(A-K_{f} C\right) \hat{x}+K_{f} y, \\
& r=0, \rho(y, r)=-\beta e^{-\alpha \alpha_{0}|y-r|}, x_{e}=G r
\end{aligned}
$$

The value of $P$ is obtained from Lyapunov method in (2). Then, the states are stated in (3). $x=\left[\begin{array}{llllllllll}z_{s} & z_{u R} & z_{u L} & \dot{z}_{s} & \dot{z}_{u R} & \dot{z}_{u L} & \phi & \dot{\phi} & V_{y} & \dot{\psi}\end{array}\right]$ (3)

\section{Optimisation of Control Strategy}

This section describes the framework for optimising the novel CNF-LQG control strategy by using NSGA-II. NSGA-II has been recently used in different areas of engineering because of its advantage of simple yet efficient non-dominance ranking procedure to yield different levels of Pareto frontiers [14]. This optimisation method is based on the natural evolution principles and genetics of population and set of non-dominated solutions can be obtained after the optimisation [15]. The main issues for the genetic algorithm are setting process of the objective functions and initial optimisation bounding the same way for the output weighting function. The details of the chosen variables, objective function formulation and constraints are described in the following [12, 16].

To design control strategies for an active anti-roll bar system, a strict trade-off exists in terms of providing a good ride comfort performance and better handling criteria by considering the roll angle response. The objective of the problem is to find the optimum values of the control design parameters that indicate good ride comfort and handling ability [17]. The optimisation problem is solved NSGA-II [18]. Eight real-coded GA variables were developed in this work to optimise the LQR and CNF controller parameters. The upper and lower limits on the parameters are set based on manual tuning of the CNF-LQG fusion control strategy by using Bryson's rule. The computational time and complexity are reduced when choosing the appropriate initial choice of the parameters and the bounder limits.

\subsection{Design Variables}

The CNF-LQG fusion control strategy for an active anti-roll bar system can be fine-tuned by choosing the suitable value of parameters, as listed in Table 1. The control parameters for the LQR controller and the CNF controller are considered for optimisation process. The values of $Q$ and $R$ are optimised for the LQR controller while the value of $\beta$ is optimised for the CNF controller. To the best of the authors' knowledge, no public domain literature is available on design optimisation of CNF controller parameters for an active anti-roll bar system by using NSGA-II. From the literature [14, 19-23], most researchers were studied the optimisation of suspension parameters or control design for suspension systems and did not study the optimisation control design for an active anti-roll bar system itself. This research gap gives rise to the idea to optimise the control design for an active anti-roll bar system. Table 1 shows the design variables, their bounds for the CNFLQG control strategy and the initial design that using intuitive method. In this study, the values of $Q, R$ and $\beta$ are observed as three design variables to be optimally found based on multiobjective optimisation of two different objective functions [17] (see in (4) and (5)).

$\left[\begin{array}{ll}\frac{1}{(0.0001)^{2}} & \frac{1}{(0.0001)^{2}}\end{array}\right] \leq Q \leq\left[\begin{array}{ll}\frac{1}{(0.1)^{2}} & \frac{1}{(0.01)^{2}}\end{array}\right]$

and

$1000 \leq$ Beta $\leq 100000$

Table 1: Design variables and bound for CNF-LQG fusion control strategy

\begin{tabular}{|c|c|c|}
\hline $\begin{array}{c}\text { Design } \\
\text { Variable }\end{array}$ & Lower-Upper & Initial Design \\
\hline$Q$ & {$\left[\begin{array}{lll}\frac{1}{(0.0001)^{2}} & \frac{1}{(0.0001)^{2}}\end{array}\right]^{-}$} & {$\left[\begin{array}{cc}\frac{1}{(0.09)^{2}} & \frac{1}{(0.09)^{2}}\end{array}\right]$} \\
\hline
\end{tabular}




\begin{tabular}{|l|c|c|}
\hline & {$\left[\frac{1}{(0.1)^{2}} \frac{1}{(0.01)^{2}}\right]$} & \\
\hline$\beta$ & $1000-100000$ & 1000 \\
\hline$R$ & $1-10$ & 3 \\
\hline
\end{tabular}

\section{Results and Discussion}

Appropriate matrices $Q$ (see in (6)) and parameter $R$ must be designed to minimise both $f_{1}$ and $f_{1}$ as much as possible, which ensures that the control system design for the LQR controller meets multiple performance indexes simultaneously while the optimised parameter of $\beta$ must be designed to achieve a comfortable ride and good handling, thereby achieving the acceptable control design of the CNF controller. The multi-objective optimisation problem with the objective functions that usually generate approaches are applied in this work. Therefore, NSGA-II is implemented to the model to obtain the Pareto-optimal solutions of matrices $Q, R$ and $\beta$ parameter.

These objective functions are considered in a Pareto optimisation process to simultaneously important trade-offs among the conflicting objectives. The evolutionary process of multi-objective optimisation is accomplished with a population size of 30 , which was chosen with probability of crossover and probability of mutation of 0.8 and 0.1 , respectively. The parameter settings of NSGA-II optimization are shown in Table 2. A total of 30 non-dominated optimum design points were obtained. Visualization tools can aid decision makers in analysing the Pareto set and selecting good solutions. Performing an accurate graphical analysis of the Pareto set points for a two-dimensional problem is easy, but it becomes more difficult for high-dimensional problems [22, 24].

Table 2: NSGA-II user defined parameters

\begin{tabular}{|c|c|}
\hline Parameter Setting & Value \\
\hline Number of generation & 300 \\
\hline Population size & 30 \\
\hline Probability of crossover & 0.8 \\
\hline Probability of mutation & 0.1 \\
\hline Distribution index in SBX & 20 \\
\hline Distribution index in polynomial mutation & 20 \\
\hline
\end{tabular}

$$
Q=\operatorname{diag}\left(\left[\frac{1}{(0.09)^{2}} \text { to } \frac{1}{(0.09)^{2}}\right],\left[\frac{1}{(0.09)^{2}} \text { to } \frac{1}{(0.09)^{2}}\right]\right)
$$

The anti-roll bar system design aims to minimise the body roll effect. Thus, this work focuses on minimising the roll angle response to optimise the controller design. Figure 2 shows the tradeoff obtained between the RMS value of roll angle during the ride performance test and the RMS value of roll angle in the handling test by using the multi-objective optimisation approach for a vehicle velocity of $70 \mathrm{~km} / \mathrm{s}$.

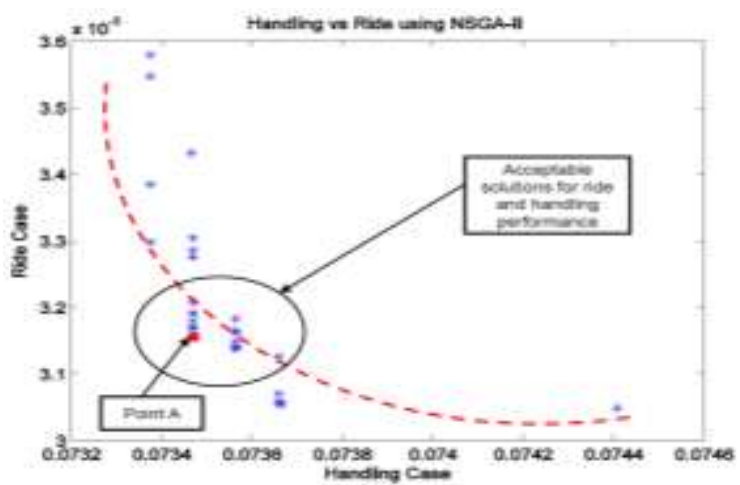

Fig. 2: RMS roll angle in handling vs RMS roll angle in ride: trade-off
NSGA-II found most solutions in the true Pareto front and its computational time is also short [25]. The figure shows that the Pareto solutions ocated close to the upper left corner have a small RMS of body roll angle in the steering input test while larger RMS body roll angle in speed bump test, which means the system achieved good handling performance but provides poor ride comfort. By contrast, the solutions near the lower right corner present worse handling but better ride comfort. Thus, in the objective space, the solutions as a result of optimisation form a Pareto optimal front, from which designers are able to make the most suitable choice that satisfies the practical requirements. Moreover, the selection of the solutions should be a compromise between the ride and handling performances.

In accordance with the Pareto chart, point $\mathrm{A}$ is applied for simulation. A solution A in central region of the trade-off is chosen for analysis. Therefore, the choice of state weighting parameters $Q$ is based on the trade-off between both situations, and gives $Q=\operatorname{diag}\left[\begin{array}{ll}\frac{1}{0.0714} & \frac{1}{0.0714}\end{array}\right], R=3.259$ and the optimal gain matrix, $K_{l q r}=$

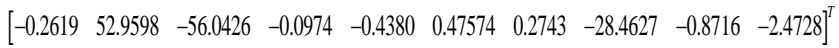

Then, the value of $\beta=1259$.

The upper and lower limits on the parameters are set based on manual or conventional tuning of the CNF-LQG fusion control strategy. The appropriate initial choice of the parameters and the limits undoubtedly reduces the computational time and complexity. The response on time domain for body roll angle and roll rate responses are shown in Figures 3 and 4 respectively. The optimisation solution offers a rather noticeable improvement results compared to the manually-tuned method. From the application point of view, both tuning process can be used. However, using optimisation method gives a multiple choice of solutions and provides the optimal parameters compared to manual tuning method. Table 3 is shown the improvement of RMS values of signal responses for CNF-LQG fusion control strategy from manual tuning method and the proposed optimization method in handling performance while Table 4 is shown the comparison of the RMS values of signal responses in ride test.
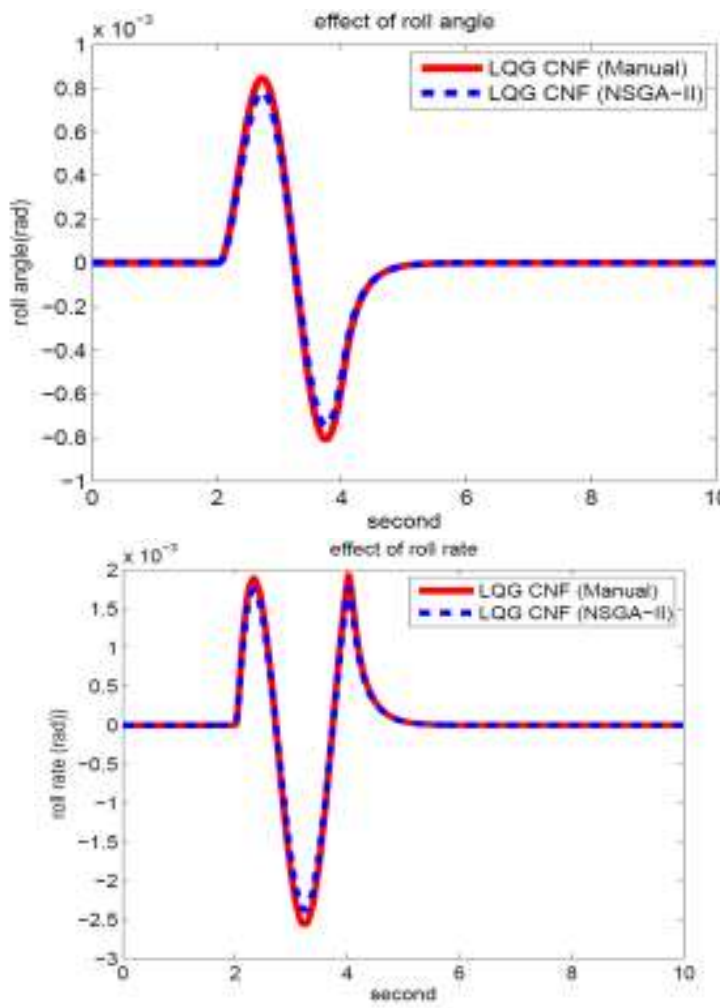

Fig. 3: single lane change (a) roll angle response; (b) roll rate response. 
Table 4: RMS values of CNF-LQG fusion with manually tuned and NSGA-II on handling test

\begin{tabular}{|c|c|c|c|}
\hline Signal Response & Manually Tuning Method & NSGA-II Optimisation & Improvement \% NSGA-II Over Manually Method \\
\hline Roll Angle & 0.00017 & 0.00015 & 6.67 \\
\hline Roll Rate & 0.00083 & 0.00082 & 1.2 \\
\hline
\end{tabular}

Figure 3 illustrates the output results of body roll angle and roll rate for the manually tuned and the optimized using NSGA-II in Single Lane Change test (handling performance). It is clearly seen that the output responses of CNF-LQG fusion control strategy using optimized method are reduced more compared to manually tuning method. The different between output responses by using these two methods are mentioned in Table 4. This results also improved for Speed Bump test.

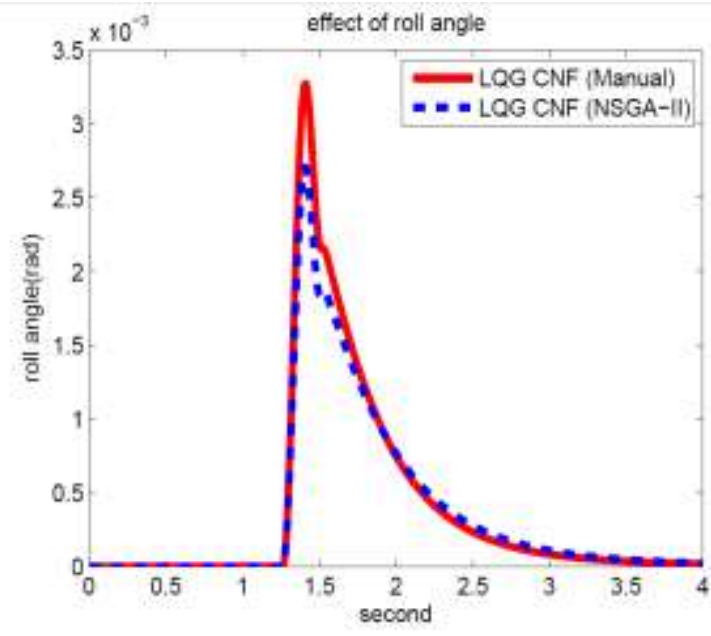

While, the output results of body roll angle and roll rate for the manually tuned and the optimized using NSGA-II in Speed Bump test (ride performance) are shown in Figure 4. It is clearly seen that the output responses of CNF-LQG fusion control strategy using optimized method are identical compared to manually tuning method. The small different between output responses by using these two methods are mentioned in Table 5.

Fig. 4: Speed bump test (a) roll angle response; (b) roll rate response.

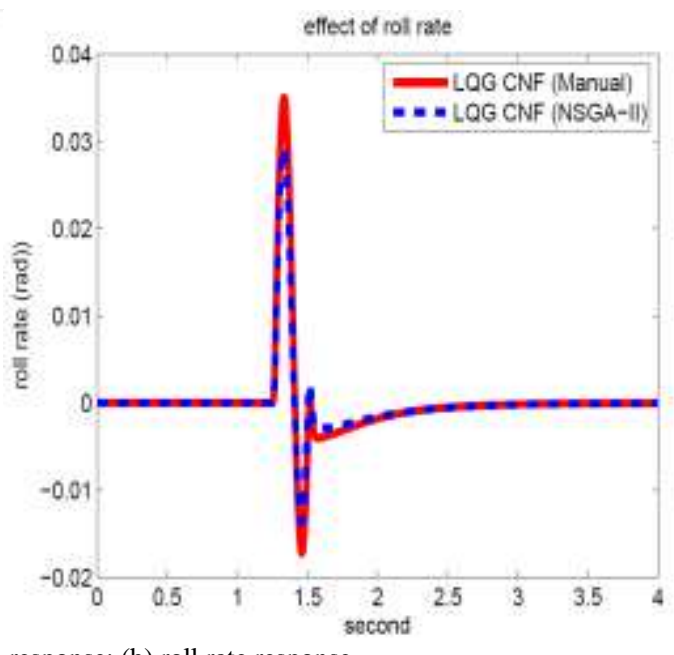

Table 5: RMS values of CNF-LQG fusion with manually tuned and NSGA-II on ride test

\begin{tabular}{|c|c|c|c|}
\hline Signal Response & Manually Tuning Method & NSGA-II Optimisation & Improvement \% NSGA-II Over Manually Method \\
\hline Roll Angle & 0.000291 & 0.000289 & 5.23 \\
\hline Roll Rate & 0.0063 & 0.0059 & 6.35 \\
\hline
\end{tabular}

\section{Conclusion}

This research discussed the optimization of the proposed control scheme namely CNF-LQG implemented base on the nonlinear vehicle model. The work on CNF-LQG fusion control design is further extended by using genetic algorithm as tuning method. This is done to give an optimal set of parameter values on controller design to meet the specified design objective. By using NSGAII gives the designer a multiple choice in selection of the solutions with regard to the system performance.

\section{Acknowledgement}

The work presented in this study is funded by the Ministry of Higher Education, Malaysia and is under a research university grant from Universiti Kebangsaan Malaysia (vote no: GGPM2017-087).

\section{References}

[1] Hac, A. 'Rollover stability index including effects of suspension design'. SAE Technical Paper, 2002.

[2] Klein, T.M. 'A statistical analysis of vehicle rollover propensity and vehicle stability'. SAE Technical Paper, 1992.

[3] Cooperrider, N.K., Thomas, T.M., Hammoud, S.A. 'Testing and analysis of vehicle rollover behavior'. SAE Technical Paper, 1990.

[4] Cimba, D., Wagner, J., Baviskar, A.: 'Investigation of active torsion bar actuator configurations to reduce vehicle body roll', Vehicle System Dynamics, 2006, 44(9), 719-736.
[5] Sampson, D.J., Cebon, D.: 'Active roll control of single unit heavy road vehicles', Vehicle System Dynamics, 2003, 40(4), 229-270.

[6] Gergely, B.: 'Application of active anti roll bar systems for enchancing yaw stability', Budapest University of Technology and Economy, 2008.

[7] Sorniotti, A., D'Alfio, N. 'Vehicle dynamics simulation to develop an active roll control system'. SAE Technical Paper, 2007.

[8] Darling, J., Hickson, L.: 'An experimental study of a prototype active anti-roll suspension system', Vehicle System Dynamics, 1998, 29(5), 309-329.

[9] Varga, B., Németh, B., Gáspár, P.: 'Design of anti-roll bar systems based on hierarchical control', Strojniški vestnik-Journal of Mechanical Engineering, 2015, 61(6), 374-382.

[10] Yim, S., Jeon, K., Yi, K.: 'An investigation into vehicle rollover prevention by coordinated control of active antiroll bar and electronic stability program', International Journal of Control, Automation and Systems, 2012, 10(2), 275-287.

[11] Krid, M., Benamar, F. 'Design and ontrol of an active anti-roll system for a fast rover'. Proceedings of the IEEE/RSJ International Conference on Intelligent Robots and Systems, 2011. pp. 274-279.

[12] Zulkarnain, N., Zamzuri, H., Sam, Y., Mazlan, S., Zainal, S. 'Improving vehicle ride and handling using lqg cnf fusion control strategy for an active antiroll bar system'. Abstract and Applied Analysis, 2014.

[13] Zulkarnain, N., Zamzuri, H., Mazlan, S.: 'Ride and handling analysis for an active anti-roll bar: Case study on composite nonlinear control strategy,' International Journal of Automotive and Mechanical Engineering, 2014, 10.

[14] Sharifi, M., Shahriari, B.: 'Pareto optimisation of vehicle suspension vibration for a nonlinear half-car model using a multiobjective genetic algorithm', Research Journal of Recent Sciences, $2012,1(8), 17-22$

[15] Zhou, R., Zolotas, A., Goodall, R. 'Lqg control for the integrated tilt and active lateral secondary suspension in high speed railway 
vehicles'. Proceedings of the 8th IEEE International Conference on Control and Automation, 2010, pp. 16-21.

[16] Zulkarnain, N., Imaduddin, F., Zamzuri, H., Mazlan, S.A. 'Application of an active anti-roll bar system for enhancing vehicle ride and handling'. Proceedings of the IEEE Colloquium on Humanities, Science and Engineering, 2012, pp. 260-265.

[17] Zamzuri, H., Zolotas, A.C., Goodall, R.M.: 'Tilt control design for high-speed trains: a study on multi-objective tuning approaches', Vehicle System Dynamics, 2008, 46(S1), 535-547.

[18] Deb, K., Pratap, A., Agarwal, S., Meyarivan, T.: 'A fast and elitist multiobjective genetic algorithm: Nsga-ii', IEEE Transactions on Evolutionary Computation, 2002, 6(2), 182-197.

[19] Khajavi, M.N., Notghi, B., Paygane, G.: 'A multi objective optimisation approach to optimize vehicle ride and handling characteristics', World Academy of Science, Engineering and Technology, 2010, 62, 580-584.

[20] Ram, A.S.S.S., Sujatha, C.: 'Multi objective optimisation of vehicle suspension design', Proceedings of the 13th International Congress on Sound and Vibration, 2006.

[21] Djukic, D., Bestle, D. 'Optimisation of damping characteristics for improved vehicle ride and handling'. Proceedings of 19th International Conference on Computer Methods in Mechanics, 2011.

[22] Sharifi, M., Shahriari, B., Bagheri, A.: 'Optimisation of sliding mode control for a vehicle suspension system via multi-objective genetic algorithm with uncertainty', Journal of Basic and Applied Scientific Research, 2012, 2(7), 6724-6729.

[23] Ahmed, F., Purdy, D. 'Controller design of active suspension system with terrain preview using evolutionary multi-objective algorithms'. Proceedings of the International Conference on Soft Computing for Problem Solving, 2012. pp. 865-876.

[24] Herrero, J., Blasco, X., Martínez, M., Ramos, C., Sanchis, J.: 'Nonlinear robust identification of a greenhouse model using multiobjective evolutionary algorithms', Biosystems Engineering, 2007, 98(3), 335-346.

[25] Kannan, S., Baskar, S., McCalley, J.D., Murugan, P.: 'Application of nsga-ii algorithm to generation expansion planning', IEEE Transactions on Power Systems, 2009, 24(1), 454-461. 